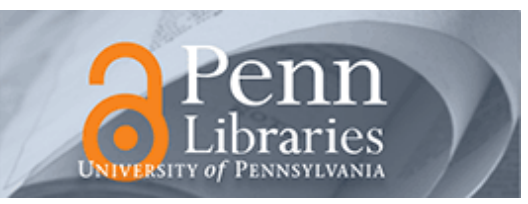

University of Pennsylvania

ScholarlyCommons

June 1996

\title{
Deformable Models with Parameter Functions for Cardiac Motion Analysis from Tagged MRI Data
}

\author{
Jinah Park \\ University of Pennsylvania \\ Dimitris Metaxas \\ University of Pennsylvania
}

Alistair A. Young

University of Auckland

Leon Axel

University of Pennsylvania, axel@oasis.rad.upenn.edu

Follow this and additional works at: https://repository.upenn.edu/hms

\section{Recommended Citation \\ Park, J., Metaxas, D., Young, A. A., \& Axel, L. (1996). Deformable Models with Parameter Functions for Cardiac Motion Analysis from Tagged MRI Data. Retrieved from https://repository.upenn.edu/hms/41}

Copyright 1996 IEEE. Reprinted from IEEE Transactions on Medical Imaging, Volume, 15, Issue 3, June 1996, pages 278-289.

This material is posted here with permission of the IEEE. Such permission of the IEEE does not in any way imply IEEE endorsement of any of the University of Pennsylvania's products or services. Internal or personal use of this material is permitted. However, permission to reprint/republish this material for advertising or promotional purposes or for creating new collective works for resale or redistribution must be obtained from the IEEE by writing to pubs-permissions@ieee.org. By choosing to view this document, you agree to all provisions of the copyright laws protecting it.

This paper is posted at ScholarlyCommons. https://repository.upenn.edu/hms/41

For more information, please contact repository@pobox.upenn.edu. 


\title{
Deformable Models with Parameter Functions for Cardiac Motion Analysis from Tagged MRI Data
}

\author{
Abstract \\ We present a new method for analyzing the motion of the heart's left ventricle (LV) from tagged magnetic \\ resonance imaging (MRI) data. Our technique is based on the development of a new class of physics- \\ based deformable models whose parameters are functions. They allow the definition of new \\ parameterized primitives and parameterized deformations which can capture the local shape variation of \\ a complex object. Furthermore, these parameters are intuitive and require no complex post-processing in \\ order to be used by a physician. Using a physics-based approach, we convert the geometric models into \\ dynamic models that deform due to forces exerted from the datapoints and conform to the given dataset. \\ We present experiments involving the extraction of the shape and motion of the LV's mid-wall during \\ systole from tagged MRI data based on a few parameter functions. Furthermore, by plotting the variations \\ over time of the extracted LV model parameters from normal and abnormal heart data along the long axis, \\ we are able to quantitatively characterize their differences.

\section{Comments} \\ Copyright 1996 IEEE. Reprinted from IEEE Transactions on Medical Imaging, Volume, 15, Issue 3, June \\ 1996, pages 278-289. \\ This material is posted here with permission of the IEEE. Such permission of the IEEE does not in any way \\ imply IEEE endorsement of any of the University of Pennsylvania's products or services. Internal or \\ personal use of this material is permitted. However, permission to reprint/republish this material for \\ advertising or promotional purposes or for creating new collective works for resale or redistribution must \\ be obtained from the IEEE by writing to pubs-permissions@ieee.org. By choosing to view this document, \\ you agree to all provisions of the copyright laws protecting it.
}




\title{
Deformable Models with Parameter Functions for Cardiac Motion Analysis from Tagged MRI Data
}

\author{
Jinah Park,* Dimitri Metaxas, Member, IEEE, Alistair A. Young, and Leon Axel, Member, IEEE
}

\begin{abstract}
We present a new method for analyzing the motion of the heart's left ventricle ( $L V$ ) from tagged magnetic resonance imaging (MRI) data. Our technique is based on the development of a new class of physics-based deformable models whose parameters are functions. They allow the definition of new parameterized primitives and parameterized deformations which can capture the local shape variation of a complex object. Furthermore, these parameters are intuitive and require no complex post-processing in order to be used by a physician. Using a physics-based approach, we convert the geometric models into dynamic models that deform due to forces exerted from the datapoints and conform to the given dataset. We present experiments involving the extraction of the shape and motion of the LV's mid-wall during systole from tagged MRI data based on a few parameter functions. Furthermore, by plotting the variations over time of the extracted $L V$ model parameters from normal and abnormal heart data along the long axis, we are able to quantitatively characterize their differences.
\end{abstract}

\section{INTRODUCTION}

A LTERATION of heart wall motion is a sensitive indicator of heart disease such as ischemia [32], which is typically caused by occlusion of a coronary vessel: The local anemia caused by the obstruction of the blood supply results in abnormal ventricular wall motion even before any significant clinical symptoms develop [35]. Moreover, abnormalities in heart wall motion are taken very seriously by physicians, because they can be life threatening injuries. However, since the heart undergoes complex motion, proper characterization of its motion still remains an open and challenging research problem.

The main difficulties in assessing heart wall motion come from two sources: 1) limitation of conventional cardiac imaging methods in terms of providing good datasets for motion studies and 2) the absence of computational techniques for automatic extraction of the three-dimensional (3-D) heart wall motion parameters in a way that is useful to physicians. Recently, the introduction of magnetic tagging [2], [39] in

Manuscript received November 18, 1994; revised November 5, 1995. The work of D. Metaxas was supported by the NSF under Grant IRI-9 309917. The Associate Editor responsible for coordinating the review of this paper and recommending its publication was J. Duncan. Asterisk indicates corresponding author

*J. Park is with the Department of Computer and Information Science at the University of Pennsylvania, Philadelphia, PA 19104-6389 USA (e-mail: jinah@graphics.cis.upenn.edu).

D. Metaxas is with the Department of Computer and Information Science at the University of Pennsylvania, Philadelphia, PA 19104-6389 USA.

A. A. Young is with the Department of Physiology at the University of Auckland, Auckland, New Zealand.

L. Axel is with the Department of Radiology at the University of Pennsylvania, Philadelphia, PA 19104-6086 USA

Publisher Item Identifier S 0278-0062(96)04233-4. magnetic resonance imaging (MRI) has provided a powerful tool to study the heart wall motion. The MR tagging methods provide temporal correspondence of material points on featureless structures like the heart wall in a noninvasive manner. Unfortunately, the tagged MR images are not easily analyzed with simple qualitative viewing, while current quantitative analysis techniques are not only time consuming, but also yield data that may not be easily interpreted for diagnosis.

A precise model that can reflect the mechanics of ventricular myocardium would provide a better understanding of the complex regional changes under pathological conditions. In addition, it is also important that the model be constructed and analyzed in close to real-time to be useful in a clinical environment. The goal of our work is to develop computational techniques for automatic extraction of the 3-D heart wall motion parameters that are not only compact, but also can give accurate descriptions of ventricular function based on tagged MR images.

\section{A. Previous Work}

In order to quantify the complicated motion of the left ventricle (LV) and to interpret its measured deformation, it is necessary to represent the LV by a model. Simple analytical shapes like spheres, ellipsoids, or cylinders are often used to approximate the shape and motion of the LV [1], [6], [13]. However, since they are formulated in terms of very few parameters, they can offer only a gross approximation to the LV motion.

Recently, 3-D surface models and associated computer vision or graphics techniques have been developed to capture the shape and motion of the inner or the outer wall of the LV from medical image data. These models are either finite element meshes derived from a polyhedron-based surface reconstruction of a stack of cross sections [8], [11], physicsbased elastic models [9], [12], [15], [20], [33], bending and stretching models [3], [31], or axisymmetrical geometric models with augmented local details [4], [18]. Some of above techniques are briefly described as follows:

- Amini and Duncan [3] developed bending and stretching thin-plate models for motion tracking of the LV wall, which allow point matching based on the amount of the bending energy. Shi et al. [31] further developed the models and presented results of the shape-based technique applied to MRI data showing motion trajectories of selected endocardial points.

- Pentland et al. [33], [28] developed a deformable model based on modal analysis, which can decompose the 
motion into modes, and applied the technique to recover the nonrigid motion of a heart from 2-D X-ray images. Extending their work, Nastar and Ayache [20] performed "spectrum" analysis in modal space for various LV's from radio-nuclide images in order to classify nonrigid motion.

- Friboulet et al. [11] constructed a polyhedral model from a set of cross section slices obtained from volumetric MRI data. The motion of the model was approximated by an affine transformation with translation, rotation, and dilation motion parameters.

- Huang and Goldgof [12] developed a spring-mass, adaptive-size mesh model and applied it to computed tomography (CT) data of a canine heart during a cardiac cycle to track the LV motion based on the displacement of corresponding model nodes at different time points.

- By adapting the deformable balloon model of Cohen and Cohen [9], McInerney and Terzopoulos [15] developed a 3-D deformable model composed of triangular $C^{1}$ finite elements based on the physics-based framework developed by Metaxas and Terzopoulos [17]. They applied it to CT data of a canine heart in order to reconstruct the LV shape at different time frames during one cardiac cycle.

- Bardinet et al. [4] estimated in detail the LV shape by using the technique of free-form deformations [29] to model local deformations in deformable superquadrics [5].

The main limitation of these techniques is that they do not provide intuitive motion parameters to describe the rigid and nonrigid motion of the LV. Most of the techniques [3], [8], [9], [12], [15], [18], [31], provide only local displacement vectors which require nontrivial post-processing to be useful to a physician, and they are good only for qualitative visualization. In contrast to these approaches, models like [4], [11] are formulated in terms of very few parameters that can offer only a gross approximation to the LV motion. There have been some attempts to characterize the LV motion with a small number of parameters [20], [33], but these parameterizations do not correspond to the geometry of the LV closely enough to provide better understanding of the LV motion.

Moreover, most techniques [3], [4], [11], [12], [15], [18], [20], [33] ignore the twisting or wringing motion of the LV known to occur during systole, since the input dataset generally does not provide temporal correspondence between frames. In order to accurately capture the heart wall motion, material points on the myocardium must be located and tracked. Therefore, techniques based on such marker-based methods, such as tagged MRI, can provide the most accurate motion of the left ventricle of a heart. They include the finite element models of Young et al. [36] and Moore et al. [19], and the multidimensional stochastic model of Denney and Prince [10]. However, these representations do not directly lend themselves to an understanding of the underlying kinematics in an intuitive way. The parameters of the models are local displacements, resulting in a large number of parameters, and therefore the physical interpretation of the parameters can be difficult. The 3-D strain tensor in [36], for example, has three normal components and three shear components, each of which may vary throughout the LV wall. In order to understand

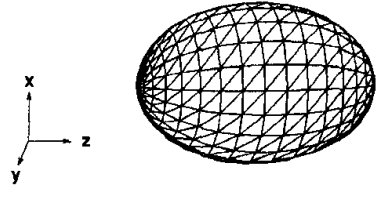

(a)

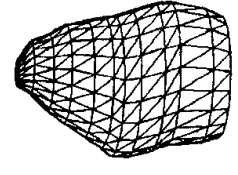

(b)
Fig. 1. (a) Superquadric ellipsoid. (b) Primitive with parameter functions.

the complex relationship between these components and other motion parameters, it is desirable to characterize the motion in terms of a small number of physical parameters without sacrificing sufficient accuracy.

To overcome the problems of the above techniques, namely the accurate estimation of the LV surface shape and motion and the extraction of parameters that can be easily interpreted by physicians, we have developed a class of deformable primitives whose global parameters are functions (see also [21], [22]). Our technique describes the time-varying shape, deformation, and motion of the $\mathrm{LV}$ in terms of a few global parameter functions, such as twisting, whose values vary locally. In this way, the complex motion of the heart is described by the same small number of parameters, whose values may vary from region to region. Furthermore, these parameters can be used by a physician directly without further complex processing.

\section{B. Proposed Model: Deformable Models with Parameter Functions}

We present a new family of parameterized deformable primitives suitable for applications where a complex shape needs to be described in terms of a small number of intuitive parameters. These deformable primitives are parameterized through a few number of parameters which are functions, and therefore each parameter's value varies across the shape of the primitives, as opposed to being constant. Through the use of appropriate parameterization, the axes of our deformable primitives can be curved. This is a major generalization compared to other parameterized primitives such as superquadrics and cylinders, commonly used in the vision literature. Even though generalized cylinders [14] allow shapes with curved axes, they do not offer a representation of shape in terms of a few parameters. Furthermore, our models can represent open parameterized shapes ${ }^{1}$ suitable for modeling the shape and motion of the LV. Fig. 1(b) shows an example of the deformable primitives with parameter functions whose shape is defined by generalizing the parametric equations of an ellipsoid shown in Fig. 1(a). The complex nonsymmetric shape in Fig. 1(b) was created by varying only six parameter functions.

While these new shape primitives can be used in many applications, this paper describes shape and motion estimation results for the LV. By incorporating the geometric definition of the models into the physics-based framework developed by Metaxas and Terzopoulos [16], [17], we create dynamic models that deform due to forces exerted from 3-D tagged datapoints, thereby causing the models to conform to the given dataset. The LV extracted parameters, plotted in parameter

\footnotetext{
${ }^{1}$ Not a closed surface, but more like a cup.
} 


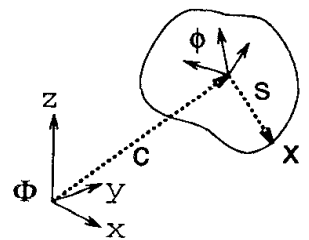

(a)

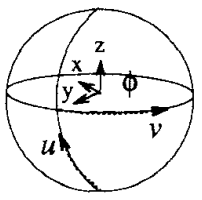

(b)
Fig. 2. (a) Model frame $\phi$ and inertial frame $\Phi$. (b) Material coordinates $(u, v)$.

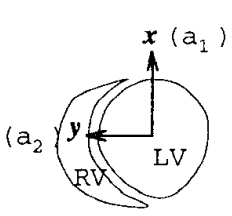

Short-axis view

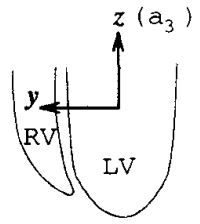

Long-axis view

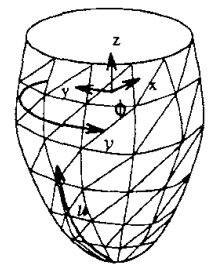

(b)
Fig. 3. Orientation of a model. (a) Short-axis view, long-axis view, (b) 3-D surface.

graphs, can then be directly used for analysis by a physician. We applied our technique to various subjects and analyzed the results of our parameter extraction. These results quantitatively verified qualitative knowledge about the LV known to physicians. Furthermore, we present a method for visualizing the model fitting results.

In the following sections, we first define the geometry of the deformable models with parameter functions (DMPF), and then describe the physics-based framework through which the geometric models are converted into dynamic models. Finally, we present experiments where we applied our technique to LV datasets from healthy volunteers and patients with hypertrophic cardiomyopathy.

\section{MODEL DEFINITION}

The class of DMPF allows the use of global parameters that can characterize an object's shape in terms of a few parameter functions. The model is a 3-D surface ${ }^{2}$ whose material coordinates $\mathbf{u}=(u, v)$ are defined in a domain $\Omega$. The positions of points on the model relative to an inertial frame of reference $\Phi$ in 3-D space are given by a vector-valued, time-varying function $\mathbf{x}(\mathbf{u}, t)=(x(\mathbf{u}, t), y(\mathbf{u}, t), z(\mathbf{u}, t))^{\top}$, where $T$ denotes transposition. We set up a noninertial, modelcentered reference frame $\phi$ and express the position of a point on a model as

$$
\mathrm{x}=\mathbf{c}+\mathbf{R s}
$$

where the center of the model, $\mathbf{c}(t)$, is the origin of $\phi$ and the rotation matrix $\mathbf{R}(t)$ gives the orientation of $\phi$ relative to $\Phi$ with a reference shape s (see Fig. 2). Thus, $\mathbf{s}(\mathbf{u}, t)$ gives the positions of points on the model relative to the model frame. Local deformations [17] are not used, since the global deformations $\mathrm{s}$ will be defined based on parameter functions capable of capturing the local variation of the LV shape.

${ }^{2}$ The model can be generalized into a volumetric model, but it is beyond the scope of this paper (see [23] and [24]).

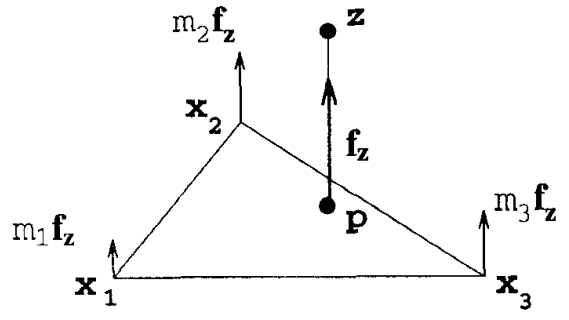

Fig. 4. Force distribution.

We define the reference shape as

$$
\mathbf{s}=\mathbf{T}\left(\mathbf{e} ; \beta_{0}(\mathbf{u}), \beta_{1}(\mathbf{u}), \cdots\right)
$$

where e can represent either a set of 3-D points in space ${ }^{3}$ or a geometric primitive $\mathbf{e}\left(\mathbf{u} ; \alpha_{0}(\mathbf{u}), \alpha_{1}(\mathbf{u}), \cdots\right)$ defined parametrically in $\mathbf{u}$ and parameterized by the variables $\alpha_{i}(\mathbf{u})$. The shape represented by $\mathrm{e}$ is subjected to the deformation $\mathrm{T}$ which depends on the deformation parameter functions $\beta_{i}(\mathbf{u})$. Although generally nonlinear, e and $\mathbf{T}$ are assumed to be differentiable $e^{4}$ so that we may compute the Jacobian of $\mathbf{s}$. $\mathbf{T}$ may be a composite sequence of primitive deformation functions $\mathbf{T}(\mathbf{e})=\mathbf{T}_{1}\left(\mathbf{T}_{2}\left(\cdots \mathbf{T}_{n}(\mathbf{e})\right)\right)$. We concatenate the deformation parameters into the vector $\mathbf{q}_{s}$

$$
\mathbf{q}_{s}=\left(\alpha_{0}(\mathbf{u}), \alpha_{1}(\mathbf{u}), \cdots, \beta_{0}(\mathbf{u}), \beta_{1}(\mathbf{u}), \cdots\right)^{\top} .
$$

The parameters $\alpha_{i}$ and $\beta_{i}$ are functions of $\mathbf{u}$, instead of constants as in our previous work [17]. This definition allows us to generalize definitions of primitives (e.g., superquadrics, cubes) and parameterized deformations (e.g., twisting), as will be shown in the following section and was demonstrated in Fig. 1(b).

\section{A. Defining the Reference Shape}

Our technique for creating primitives with parameter functions can be applied to any parametric primitive, by replacing its constant parameters with differentiable parameter functions. For example, we define a generalized primitive $\mathbf{e}=$ $\left(e_{1}, e_{2}, e_{3}\right)^{\top}$ to be used for modeling the $\mathbf{L V}$ wall as follows:

$$
\begin{aligned}
\mathbf{e} & =\mathbf{e}\left(\mathbf{u} ; a_{0}(\mathbf{u}), a_{1}(\mathbf{u}), a_{2}(\mathbf{u}), a_{3}(\mathbf{u})\right) \\
& =a_{0}(\mathbf{u})\left(\begin{array}{c}
a_{1}(\mathbf{u}) \cos u \cos v \\
a_{2}(\mathbf{u}) \cos u \sin v \\
a_{3}(\mathbf{u}) \sin u
\end{array}\right)
\end{aligned}
$$

where $-\pi / 2 \leq u \leq \pi / 4,-\pi \leq v<\pi, a_{0}(\mathbf{u})>0$, and $0 \leq a_{1}(\mathbf{u}), a_{2}(\mathbf{u}), a_{3}(\mathbf{u}) \leq 1$. This primitive is created from an ellipsoid primitive $\mathbf{e}_{e}$

$$
\mathrm{e}_{e}=a_{0}\left(\begin{array}{c}
a_{1} \cos u \cos v \\
a_{2} \cos u \sin v \\
a_{3} \sin u
\end{array}\right)
$$

where $-\pi / 2 \leq u \leq \pi / 2,-\pi \leq v<\pi, a_{0}>0$, and $0 \leq a_{1}, a_{2}, a_{3} \leq 1$, by replacing its constant parameters with parameter functions. $a_{0}$ is a scale parameter and $a_{1}, a_{2}$, and

\footnotetext{
${ }^{3}$ In that case, the material coordinates $\mathbf{u}$ coincide with the Cartesian space in which the 3-D points are expressed.

${ }^{4}$ In the case where $\mathrm{e}$ is a set of points, the above assumption does not apply.
} 


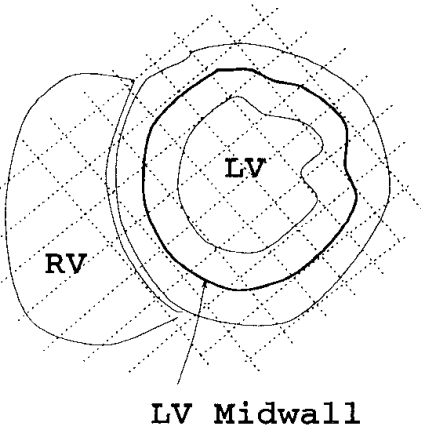

(a)

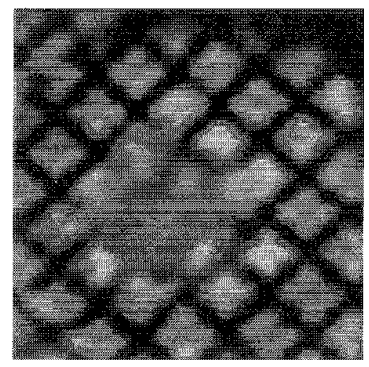

(b)

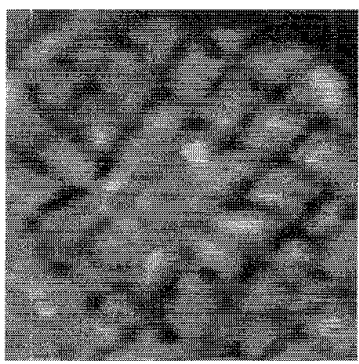

(c)

Fig. 5. SPAMM images: (a) Short-axis view, (b) early systole, and (c) end systole.

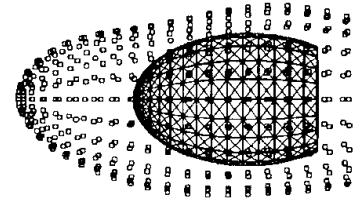

(a)

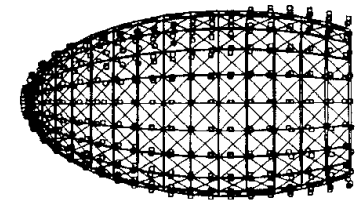

(b)

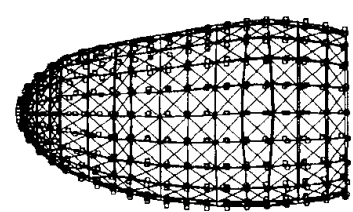

(c)

Fig. 6. Fitting a model to data of the LV midwall from a normal heart.

$a_{3}$ are the aspect ratio parameters along the $x-, y$ - and $z$-axes, respectively. Note that the ranges of the $u$ and $v$ parameters for our generalized primitive (1) are restricted to a subset of those for an ellipsoid primitive defined by (2), in order to construct an open parameterized primitive. The orientation of a model used for our application is schematically drawn in Fig. 3. The model-centered reference frame $\phi$ is chosen at the center of the $\mathrm{LV}$ with the $y$ axis pointing toward the right ventricle (RV). The material coordinates $\mathbf{u}=(u, v)$ are depicted in Fig. 3(b), where $u$ runs from the apex to the base of the LV, and $v$ starts and ends at the point where the midseptum is located. Note that as shown in Fig. 3(b), the model is tessellated into planar triangular elements.

Our formulation of deformations with continuous parameter functions is general and can be applied to any underlying shape e. For our application, we will define a model which includes parameterized twisting and axis offset deformations.

Given a primitive, e, we define parameterized twisting along the model axis $z$, which results in the global shape $\mathbf{s}_{t}=\left(s_{1}, s_{2}, s_{3}\right)^{\top}$

$$
\begin{aligned}
\mathbf{s}_{t} & =\mathbf{T}_{t}(\mathbf{e} ; \tau(\mathbf{u})) \\
& =\left(\begin{array}{c}
e_{1} \cos (\tau(\mathbf{u}))-e_{2} \sin (\tau(\mathbf{u})) \\
e_{1} \sin (\tau(\mathbf{u}))+e_{2} \cos (\tau(\mathbf{u})) \\
e_{3}
\end{array}\right)
\end{aligned}
$$

where $\tau(\mathbf{u})$ is the twisting parameter function along the $z$ axis. Finally, we apply offset deformations which allow the axis to be nonstraight in the $x$ and $y$ directions. In this way we can recover the LV shape more accurately. The resulting reference shape $\mathbf{s}$ is expressed as follows:

$$
\begin{aligned}
\mathbf{s} & =\mathbf{T}_{o}\left(\mathbf{s}_{t} ; e_{1_{\diamond}}(\mathbf{u}), e_{2_{o}}(\mathbf{u})\right) \\
& =\left(\begin{array}{c}
s_{1}+e_{1_{\diamond}}(\mathbf{u}) \\
s_{2}+e_{2_{o}}(\mathbf{u}) \\
s_{3}
\end{array}\right)
\end{aligned}
$$

where $e_{1_{\circ}}(\mathbf{u})$ and $e_{2_{\diamond}}(\mathbf{u})$ are axis-offset parameter functions in the $x$ and $y$ directions, respectively.

For the applications presented here, we have defined seven parameter functions for our models. ${ }^{5}$ Therefore, the deformation parameter vector $\mathbf{q}_{s}$ becomes

$$
\mathbf{q}_{s}=\left(a_{0}(\mathbf{u}), a_{1}(\mathbf{u}), a_{2}(\mathbf{u}), a_{3}(\mathbf{u}), \tau(\mathbf{u}), e_{1}(\mathbf{u}), e_{2_{o}}(\mathbf{u})\right)^{\top} .
$$

The set of these parameters was sufficient to characterize the LV shape and motion for our application. We have also experimented with deformations such as oblique (nonplanar) bending (see [21]), but did not find them clinically relevant given our data. Note that the above defined parameters are carefully chosen so that they represent independent degrees of freedom.

Without loss of generality, we will assume that all our model parameters are functions of $u$ [i.e., $\alpha_{i}(\mathbf{u})=\alpha_{i}(u), \beta_{i}(\mathbf{u})=$ $\beta_{i}(u)$ ], allowing them to vary from the apex to the base of the LV. We may define the parameters as functions of circumferential position (i.e., v) as well. However, it was not necessary for the experiments provided in this paper. The choice of the parameter functions depends on the application. For the applications in this paper we assume that the parameter functions are piecewise linear along $u$, so we do not impose any shape continuity constraints on the LV shape and motion. In other words, the model deforms based on the motion dictated by the dataset and not on the imposition of constraints such as artificial elastic properties. It is difficult to obtain elastic properties for the myocardium, since they may vary among hearts and cannot be measured from MRI data.

\footnotetext{
${ }^{5}$ Refer to Section IV-B and Fig. 7 for the interpretation of each parameter function.
} 


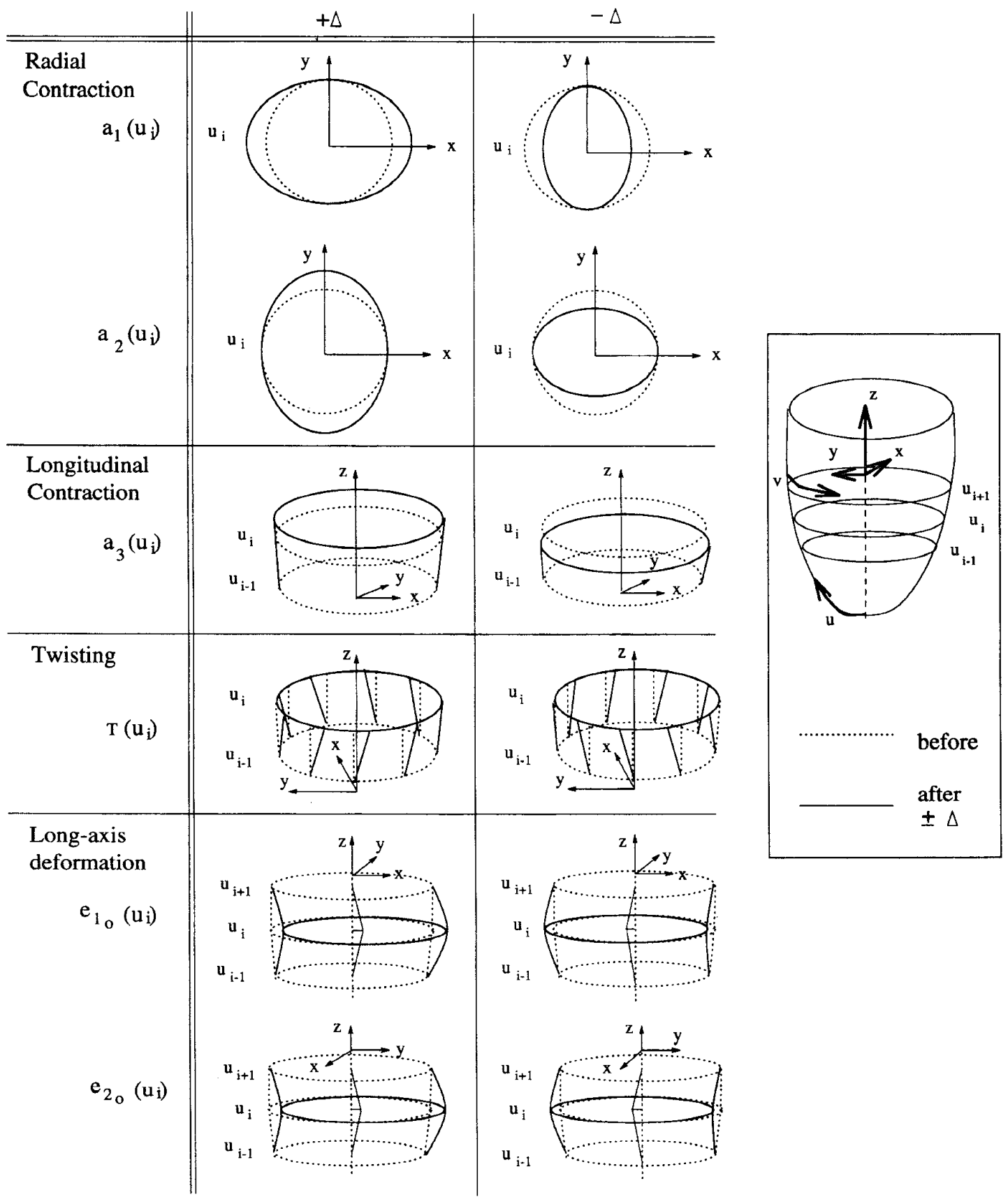

Fig. 7. Effect of changing the value of each parameter function.

III. Kinematics AND DynamiCs of THE System

The velocity of points on the model is given by [30]

$$
\begin{aligned}
\dot{\mathbf{x}} & =\frac{d(\mathbf{x})}{d t}=\frac{d(\mathbf{c}+\mathbf{R} \mathbf{s})}{d t} \\
& =\dot{\mathbf{c}}+\dot{\mathbf{R}} \mathbf{s}+\mathbf{R} \dot{\mathbf{s}} \\
& =\dot{\mathbf{c}}+\mathbf{B} \dot{\theta}+\mathbf{R} \dot{\mathbf{s}}
\end{aligned}
$$

where $\theta=\left(\cdots, \theta_{i}, \cdots\right)^{\top}$ is the vector of rotational coordinates of the model and $\mathbf{B}=\left[\cdots \partial(\mathbf{R s}) / \partial \theta_{i} \cdots\right]$.
Furthermore

$$
\dot{\mathbf{s}}=\left[\frac{\partial \mathrm{s}}{\partial \mathbf{q}_{s}}\right] \dot{\mathbf{q}}_{s}=\mathbf{J} \dot{\mathbf{q}}_{s}
$$

where $\mathbf{J}$ is the Jacobian of the primitive function. Therefore, we can write

$$
\dot{\mathbf{x}}=\left[\begin{array}{lll}
\mathbf{I} & \mathbf{B} & \mathbf{R J}
\end{array}\right] \dot{\mathbf{q}}=\mathbf{L} \dot{\mathbf{q}}
$$

where $\mathbf{L}$ is the model's Jacobian matrix which maps the model's parameter space into 3-D space, and $\mathrm{q}=$ $\left(\mathbf{q}_{c}^{\top}, \mathbf{q}_{\theta}^{\top}, \mathbf{q}_{s}^{\top}\right)^{\top}$ is the vector of the model's degrees of 


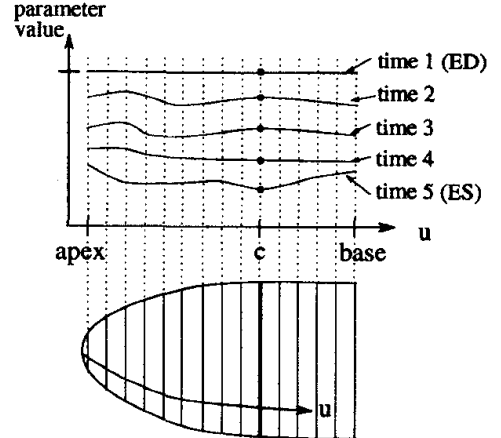

Fig. 8. Interpretation of parameter graphs.

freedom. $\mathbf{q}_{c}=\mathbf{c}$ is the translation vector, and $\mathbf{q}_{\theta}$ is the vector of the model's rotational parameters. Note that we represent the rotation vector $\mathbf{q}_{\theta}$ as a quaternion [7].

The goal of fitting the model to datapoints is to recover the vector $\mathbf{q}$. This is achieved by carrying out the fitting procedure in a physics-based way-by enabling the data to apply traction forces onto the surface of the model [34].

\section{A. Simplified Lagrange Equations of Motion}

We can make the model dynamic in $\mathbf{q}$, in general, by introducing mass, damping, and a deformation strain energy [16], [17]. The governing Lagrange equations of motion are second-order differential equations given as follows

$$
\mathbf{M} \ddot{\mathbf{q}}+\mathbf{D} \dot{\mathbf{q}}+\mathbf{K q}=\mathbf{g}_{q}+\mathbf{f}_{q}
$$

where $\mathbf{M}, \mathbf{D}$, and $\mathbf{K}$ are the mass, damping, and stiffness matrices, respectively. $\mathbf{g}_{q}$ are inertial forces arising from the dynamic coupling between the local and global degrees of freedom, and $\mathbf{f}_{q}(\mathbf{u}, t)$ are the generalized external forces associated with the degrees of freedom of the model. In shape estimation problems [16], it makes sense to simplify the motion equations while preserving useful dynamics by setting the mass density to zero to obtain

$$
\mathbf{D} \dot{\mathbf{q}}+\mathbf{K q}=\mathbf{f}_{q} .
$$

The above equation yields a model that has no inertia and comes to rest as soon as all the applied forces equilibrate or vanish. We use $\mathrm{D}$ as a stabilizing factor only, and we do not impose any physical damping which cannot be measured from our data. Therefore, we assume that $\mathbf{D}$ is diagonal and constant over time.

Given that the datapoints from medical images are relatively accurate, and we want to avoid undesired smoothing caused by the model, we further simplify (4) by introducing null stiffness to the global parameters of our model (this is like a plastic deformation where there is no resistance to deformation). Finally, the resulting equation of motion is

$$
\mathbf{D} \dot{\mathbf{q}}=\mathbf{f}_{q} .
$$

For fast interactive response, a first-order Euler method [26] is employed to integrate (5).

\section{B. Model Force Computation} [16]

The generalized forces, $\mathbf{f}_{q}$, are computed using the formula

$$
\mathbf{f}_{q}=\int \mathbf{L}^{\top} \mathbf{f} d u
$$

These forces modify the components of $\mathbf{q}$, where $\mathbf{f}(\mathbf{u}, t)$ is the 3-D force distribution applied to the model. Approximating each triangular element of the model with a plane, we determine the closest point $\mathbf{p}$ on the model for each given datapoint $\mathbf{z}$. The force that $\mathbf{z}$ exerts on the model is computed from

$$
\mathbf{f}_{z}=\gamma(\mathbf{z}-\mathbf{p})
$$

where $\gamma$ is the strength of the force. We then linearly distribute $\mathrm{f}_{z}$ to the nodes $\mathrm{x}_{1}, \mathrm{x}_{2}$, and $\mathrm{x}_{3}$ of the associated triangular element based on the formula

$$
\mathbf{f}_{\mathbf{x}_{i}}=m_{i} \mathbf{f}_{z}, \quad i=1,2,3
$$

where the $m_{i}$ are computed from the solution of the following linear system

$$
\sum_{i} m_{i} \mathbf{x}_{i}=\mathbf{p}
$$

and their sum is such that

$$
\sum_{i} m_{i}=1.0
$$

Therefore the following equation is also true

$$
\sum_{i} m_{i} \mathbf{f}_{z}=\mathbf{f}_{z}
$$

Intuitively, each of the $m_{i}$ 's is a weight given to each element node and the vector $p$ is the location of the center of mass of the element.

Since the tagged dataset provides correspondence over time of individual 3-D points, we apply the force distribution algorithm only once for the initial frame. In subsequent frames, the corresponding points will exert a force to the same point on the model as computed in the first frame. In this way we can recover the $\mathrm{LV}$ twisting motion.

\section{Model Fitting to Tagged Data}

\section{A. Data Acquisition}

We apply our technique to SPAMM data sequences from two normal hearts and two abnormal hearts with hypertrophic cardiomyopathy. The data were obtained from the Department of Radiology at the University of Pennsylvania and were collected during the LV systole over five intervals. When a saturation pulse sequence is applied prior to imaging, the amplitude of the magnetization varies spatially, in a sinusoidallike fashion. At the minima of this sinusoidal-like variation of the magnetization, dark lines appear. If we continue to image the tissue after the saturation pulse sequence is applied, we can see those dark lines move, allowing us to track the motion of the underlying tissue. Fig. 5(b) and (c) shows short-axis views of an LV at early systole and toward end-systole, respectively. One drawback of the current MR tagging technique is that the 
tracking is possible only during systole or diastole at one time (i.e., not for a complete heart cycle), due to the decay of the magnetization signal over time as can be observed in Fig. 5.

The SPAMM technique provides data throughout the heart wall. However, since our modeling technique is surface based, we chose to fit the LV mid-wall motion since this is most accurately defined by the SPAMM imaging technique. The datasets used in the current study comprised 400 material points each, whose position described the geometry and motion of the mid-wall surface of the LV during systole. The mid-wall data was obtained from 3-D reconstructions of the geometry and motion of the LV performed previously [36]-[38]. Briefly, the locations of the inner and outer boundaries of the LV were defined manually on each frame, and the tags were tracked using a semiautomatic procedure based on snakes [38]. A high-order finite-element model was fitted to the locations of the inner and outer LV contours at end-diastole. This model comprised 16 3-D finite elements (40 nodes) with bicubic interpolation in the circumferential and longitudinal directions and linear interpolation in the transmural direction [36]. The model then deformed to match the displacements of the tracked tag data, based on a least-squares based approach. The midwall surface of the model was sampled to provide a set of material points equally spaced around the surface. This data was then used as input for the experiments presented in the following section.

\section{B. Model Fitting to Tagged Datapoints over Time}

Given a set of tagged 3-D datapoints from the LV mid-wall during systole, we first fit a model to the initial time frame (i.e., end-diastole). This is done by first overlaying a simple model, which resembles an ellipsoid, onto the data. Initially, the model frame is placed at the center of mass of the datapoints (square dots in Fig. 6). The forces acting on the model will cause it to translate and to rotate, to find a suitable position and orientation as shown in Fig. 6(a). Then the nodes on the model are pulled toward the datapoints by the generalized forces, described in Section III, concurrently updating all the deformation parameter values. When all applied forces equilibrate or vanish, or the error of fit (the distance between a datapoint and the model surface) diminishes below an acceptable threshold, the model comes to rest. Fig. 6(c) shows our model fitted to the data. Fig. 6(b) shows, for demonstration purposes only, a model with constant parameters (an ellipsoid) fitted to the data. The inadequacy of such a model to obtain an accurate fit is obvious, and we can easily observe the improvement of fitting in Fig. 6(c) compared with Fig. 6(b). The length of the LV is approximately $100 \mathrm{~mm}$. The average distance error of fit for Fig. 6(b) is $1.3 \mathrm{~mm}$ and the rms error for Fig. $6(\mathrm{~b})$ is $0.83 \mathrm{~mm}$, while the average distance error for Fig. 6(c) is $0.86 \mathrm{~mm}$ and the rms error for Fig. $6(\mathrm{c})$ is $0.48 \mathrm{~mm}$.

The model fitted to the data from the first time frame is then used as the initial shape to fit the data from the second time frame. Then, the model fitted to the second time frame is used as the initial shape to fit the data from the third time frame, likewise, for the subsequent frames. Since the datapoints in subsequent time frames are tagged, the model deforms to the
TABLE I

PARAMETERS

\begin{tabular}{cc}
\hline Parameters & Representation \\
\hline$a_{1}(u), a_{2}(u)$ & radial contractions \\
$a_{3}(u)$ & longitudinal contraction \\
$r(u)$ & twisting about the long axis \\
$e_{1_{0}}: e_{2}(u)$ & long-axis deformation \\
\hline
\end{tabular}

corresponding points in the next time frame. Fig. 9(a) shows the model shown in Fig. 6(c) from a different viewpoint, and Fig. 9(b)-(e) shows the model fitted to subsequent time frames during systole.

As described in Section II, our model is defined by six deformation parameter functions in addition to global translation and rotation. In the process of fitting the model to datapoints from subsequent time frames, the global translation is kept constant, because the amount of translation of the model frame depends on where the center of mass of the datapoints is located at each frame. It may be arbitrary and may result in false estimation of the deformation parameters especially the longitudinal contraction of the LV. But if there is a significant translation in $x y z$, we can recover it, since it will be reflected in the $e_{1_{o}}(u), e_{2_{o}}(u)$, and $a_{3}(u)$ parameters. We compute the global rotation of the model frame before estimating the model deformations. In this way, we can estimate separately the global rotation and the twisting deformations.

The scaling parameter function $a_{0}(u)$ is also kept unchanged during the fitting of subsequent time frames so that the scaling variation is captured by the aspect ratio parameters $\left(a_{1}(u), a_{2}(u)\right.$, and $\left.a_{3}(u)\right)$ over time. Therefore, our model requires only six deformation parameter functions in order to characterize the LV motion, as summarized in Table I, and a quaternion vector to represent the rotation of the model.

Deformation Parameters: The parameter functions we use in our experiments can be interpreted intuitively without any further complex processing. Since our model is in a normalized scale, we utilize the scaling parameter function $a_{0}(u)$, which is constant over $u$. Once the value of $a_{0}(u)$ is set for the first time frame (i.e., end-diastole), it does not change during subsequent time frames. $a_{1}(u), a_{2}(u)$, and $a_{3}(u)$ are the aspect ratios along the $x-, y-$, and $z$-axes, respectively. Since the short-axis views of the LV lie in the $x y$ plane, the changes in $a_{1}(u)$ and $a_{2}(u)$ over time will capture the radial contraction of the LV. Likewise, the changes in the aspect ratio along the $z$ axis [i.e., $\left.a_{3}(u)\right]$ will capture the longitudinal contraction of the LV. The twisting parameter $\tau(u)$ is defined about the $z$ axis which coincides with the long axis of the LV. The axis offset parameters $e_{1_{0}}(u)$ and $e_{2_{o}}(u)$ allow the long axis to be nonstraight in the $x$ and $y$ directions, in order to capture more accurately the shape variation over time of the LV.

Fig. 7 demonstrates the effect of changing the value of each parameter function at a particular point $u_{i}$ along $u .+\Delta$ and $-\Delta$ denote an increase or decrease, respectively, in the value of the relevant parameter function at $u_{i}$. The dotted lines denote the initial shape of the deformable model at $u_{i}$, while the solid line denotes its shape after changing the value of the relevant parameter function. 


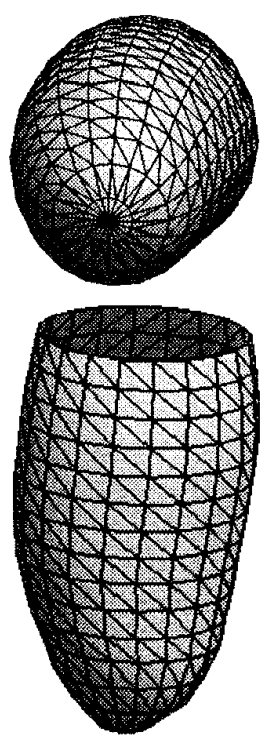

(a)
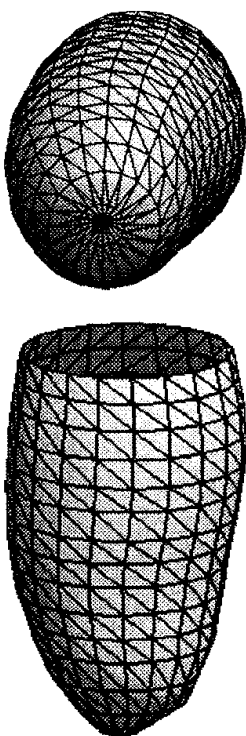

(b)
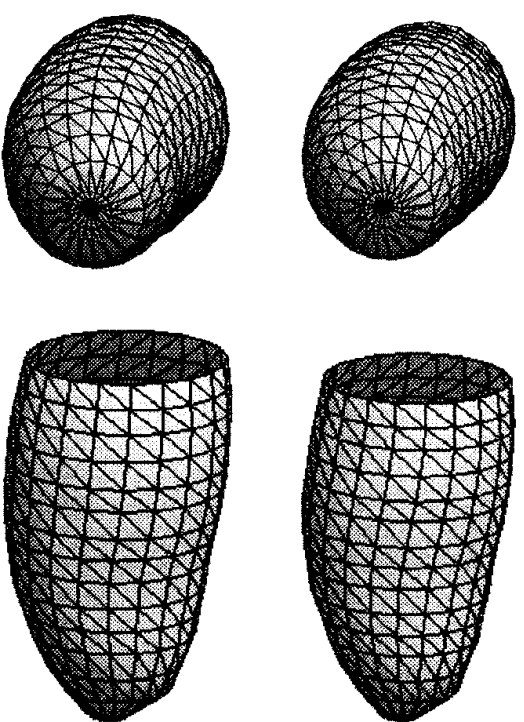

(c)

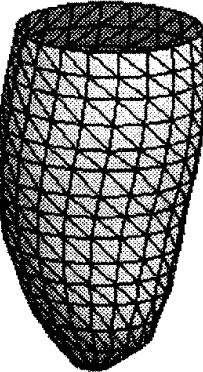

(d)
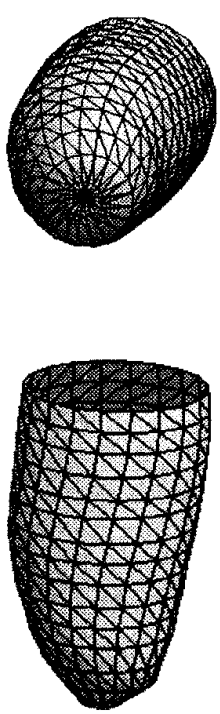

(e)

Fig. 9. Model fitted to SPAMM data (LV mid-wall) from a normal heart during systole: (a) time 1 (ED), (b) time 2, (c) time 3, (d) time 4, and (e) time 5 (ES).

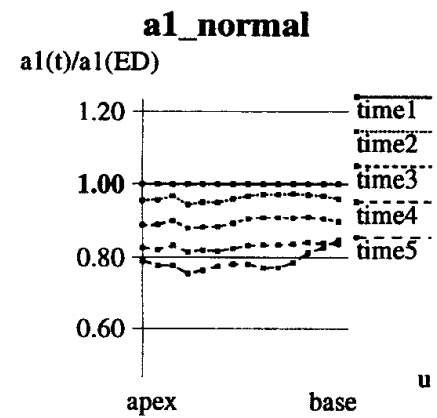

(a)

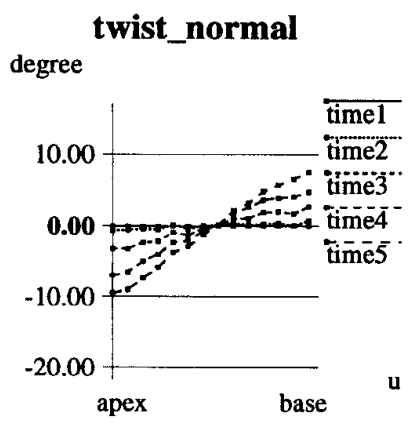

(d)

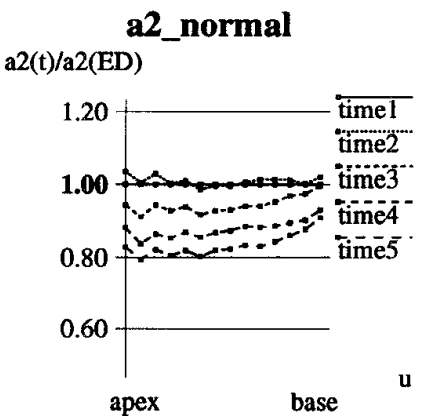

(b)

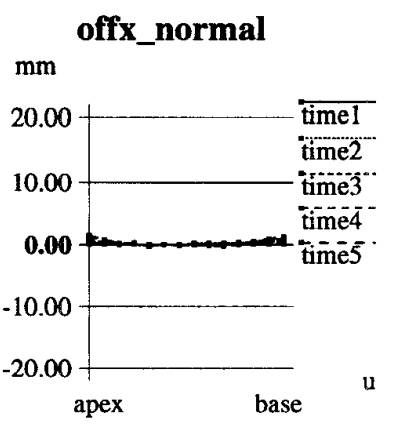

(e)

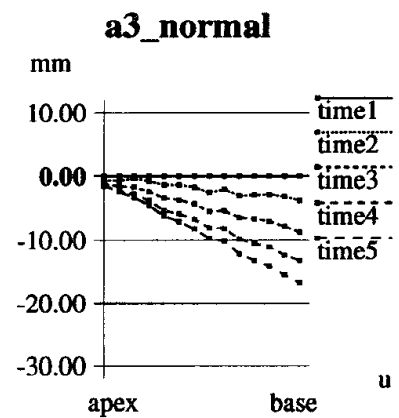

(c)

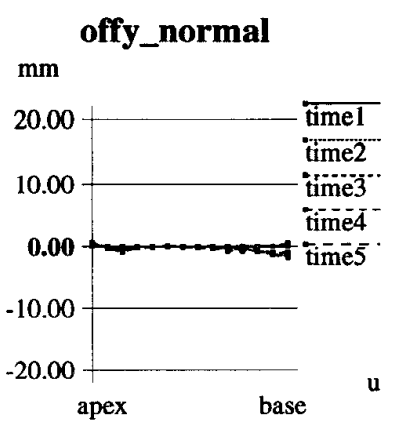

(f)

Fig. 10. Extracted model parameters as functions of $u$ for the normal heart.

\section{LV Fitting Results}

Fig. 8 depicts how we plot the parameter functions in the graphs shown in Figs. 10 and 12. The parameter values are plotted as a function of $u$, which varies from the apex to the base of an $\mathrm{LV}$, for each time frame $t(t=1 \cdots 5)$. In this way, we can observe their variation along the long axis of the LV (u) for each time frame. As an example, in Fig. 8 we show how to observe the parameter value changes during systole at the long axis location $u=c$.
1) Normal LV's in Systole: Fig. 9 shows two different views of the model fitting results to data from a normal heart taken over five time sequences during systole [from end-diastole $(t=1)$ to end-systole $(l=5)$ ]. We can easily observe the contraction as well as twisting of the model. Using the parameter functions, we can quantify the observed variations along the long axis of the LV over time.

Fig. 10 we plots some of the extracted model parameter functions over the five time frames for the normal heart. 


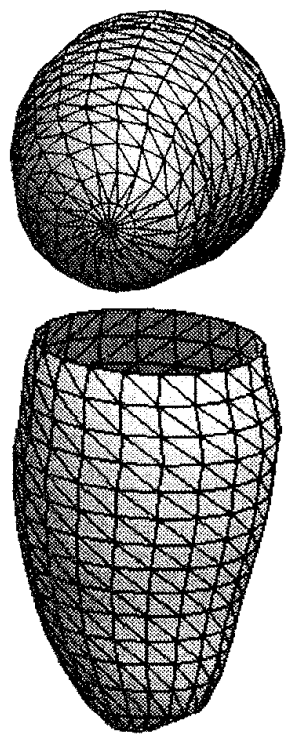

(a)

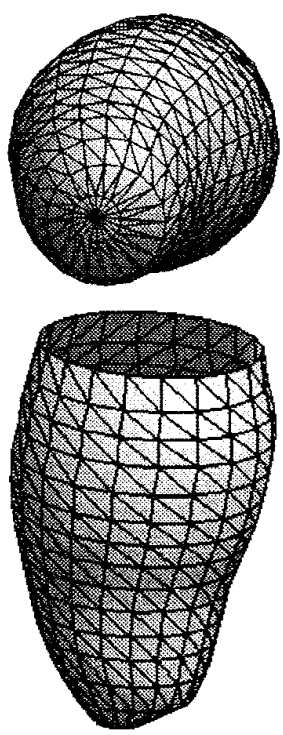

(b)
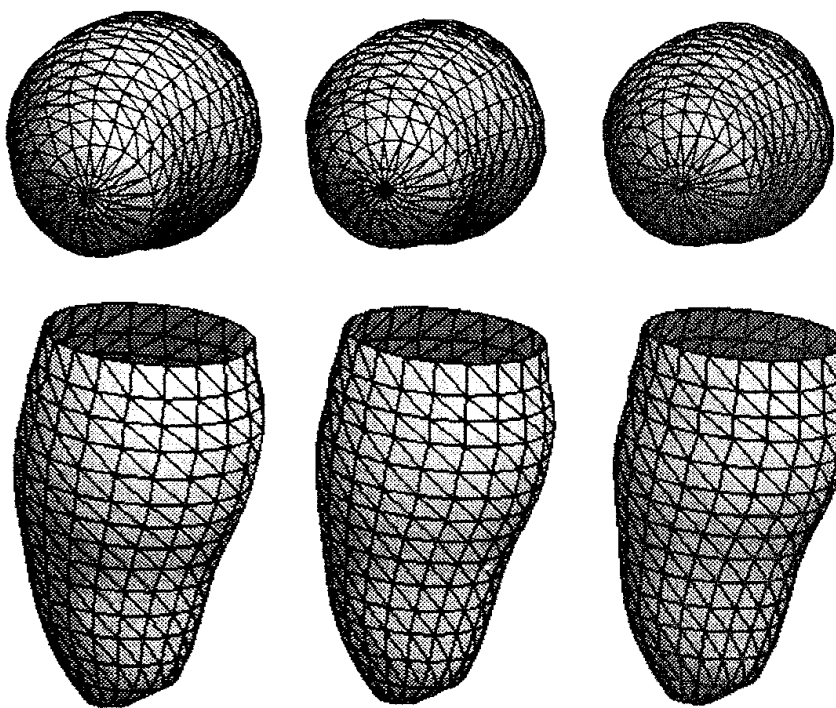

(c)

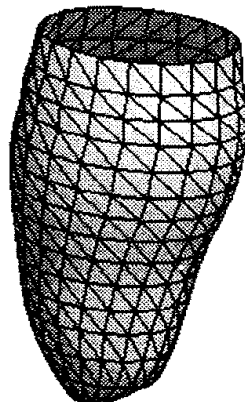

(d)

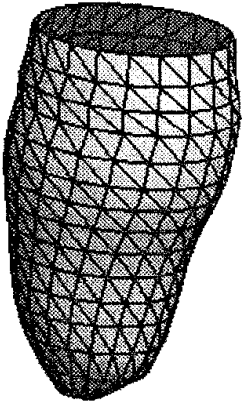

(e)

Fig. 11. Model fitted to SPAMM data from an abnormal heart during systole: (a) time 1 (ED), (b) time 2, (c) time 3, (d) time 4, and (e) time 5 (ES).

a1_abnormal $\operatorname{al}(\mathrm{t}) / \mathrm{a} 1(\mathrm{ED})$

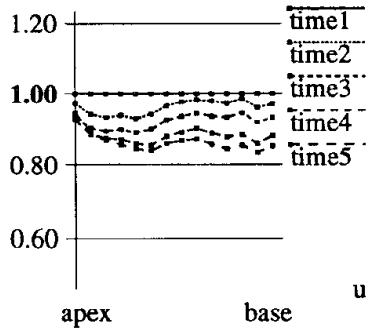

(a)

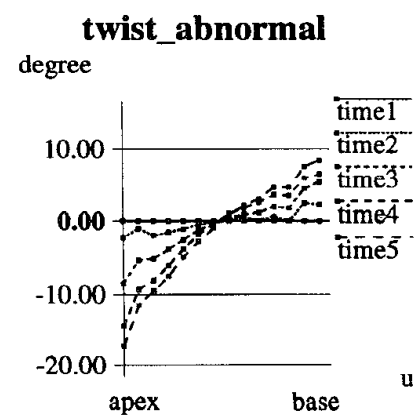

(d)

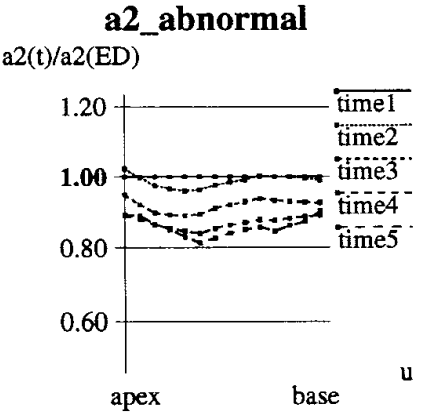

(b)

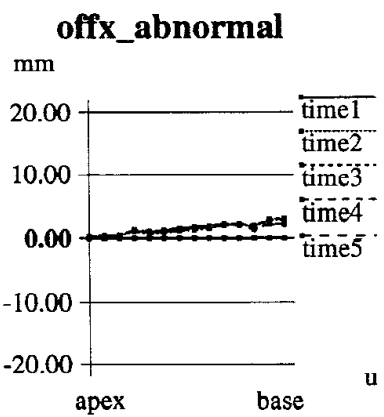

(e)

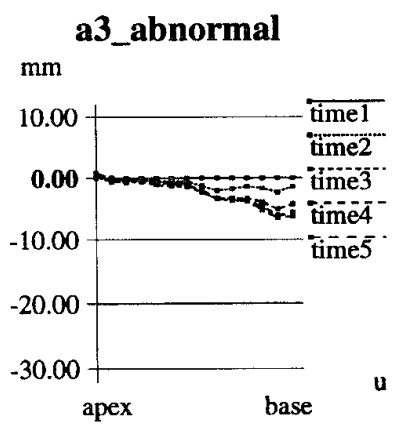

(c)

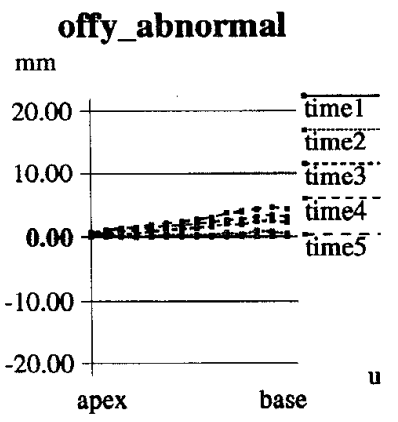

(f)

Fig. 12. Extracted model parameters as functions of $u$ for the abnormal heart.

Fig. 10(a) and (b) shows the plots of the model's parameter functions $a_{1}(u)$ and $a_{2}(u)$, which are associated with its length in the $x$ and $y$ directions, respectively. For each frame we plot the percentage of change in each parameter function during frame $t=2 \cdots 5$, with respect to its value at the initial frame $(t=1)$. Fig. 10(c) shows plots of the displacement of the length along the $z$ direction computed from the parameter function $a_{3}(u)$. Fig. 10(d) shows plots of the model's twisting parameter function $\tau(u)$. Finally, Fig. 10(e) and (f) shows plots of the model's long-axis deformation parameters $e_{1_{o}}(u)$ and $e_{2_{o}}(u)$, respectively.

From these graphs, we can quantify the shape and motion of the LV during its systole. For example, by studying the graphs of $a_{1}(u)$ and $a_{2}(u)$ [Fig. 10(a) and (b)], we can conclude that the magnitude of contraction in the radial direction (i.e., along the $x$ and $y$ axes) during systole is approximately 

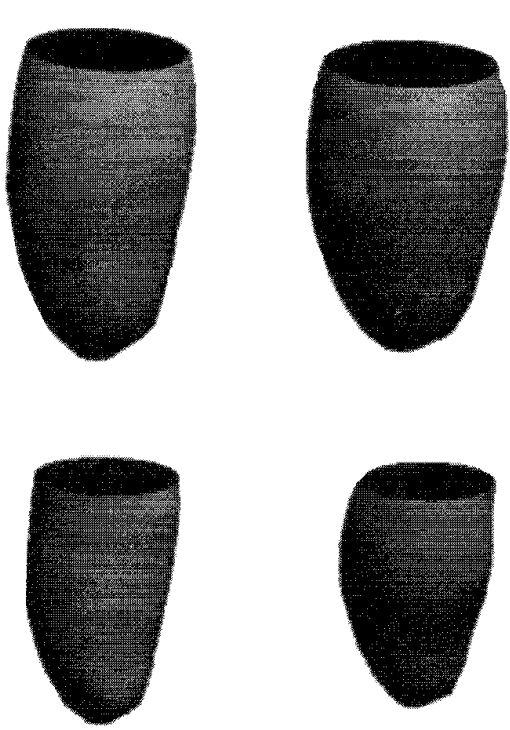

(a)

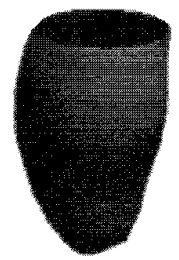

(b)
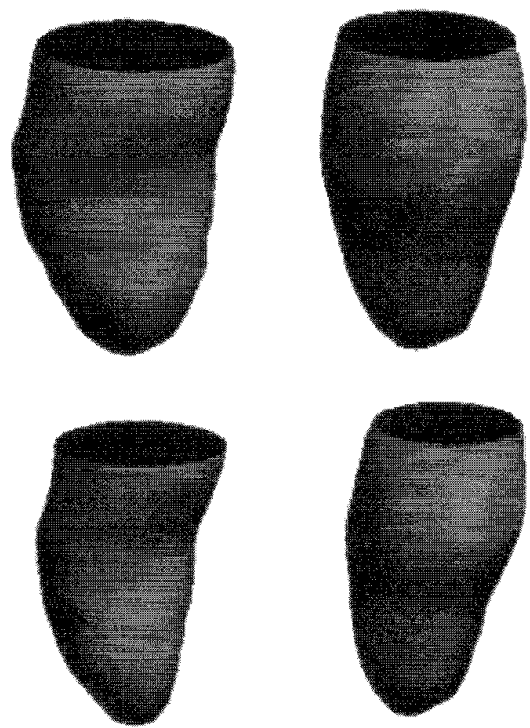

(c)

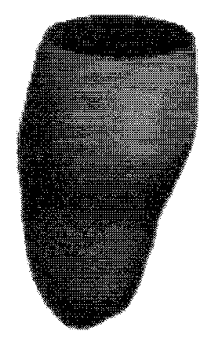

(d)

Fig. 13. Models fitted to four subjects (top: at end-diastole, bottom: at end-systole): (a) volunteer 1, (b) volunteer 2 , (c) patient 1 , and (d) patient 2 .

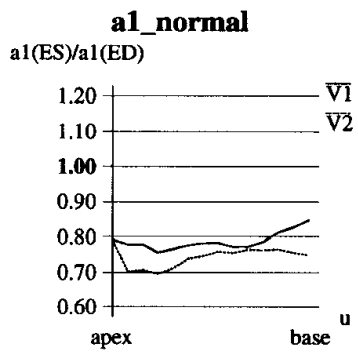

(a)

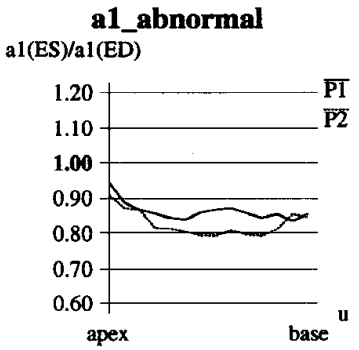

(b)

Fig. 14. Extracted LV parameter $a_{1}(u)$ at end-systole: (a) normal hearts, and (b) abnormal hearts.

$20-25 \%$. While the graph of $a_{1}$ shows uniform contraction along the long axis of the LV, the graph of $a_{2}$ shows less contraction toward the base of the LV making the base look more elliptical. This result supports clinical study findings where more stress is exerted at the apex during the LV motion, and also there is an increased similarity of the LV base shape to an ellipse, during systole. We measure from the graph shown in Fig. 10(c), that the total displacement along the $z$ axis, which corresponds to contraction along the $z$ axis, is approximately $18 \mathrm{~mm}$, where the length of the $\mathrm{LV}$ is approximately 75 $\mathrm{mm}$. Therefore, the contraction along the $z$ axis known as longitudinal contraction is approximately $24 \%$ for this LV. From the graph in Fig. 10(d), we can quantify the twisting motion of the LV during systole to approximately $18^{\circ}$. The graph shows that there is a small amount of twisting in early systole with gradual increases toward end-systole. Finally, from the long-axis deformation parameters (or axis offset parameters) shown in Fig. 10(e)-(f), we observe that there are only slight deformations and most of the deformation appears toward its apex and base, capturing a bending motion of the long axis. By having the graphs of the parameter functions plotted next to the animation, we can quantify and easily

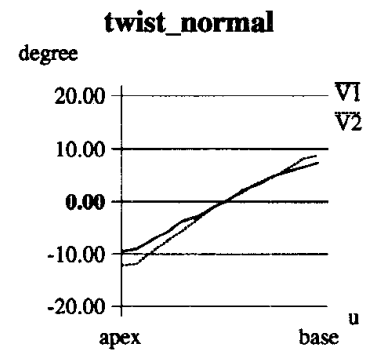

(a)

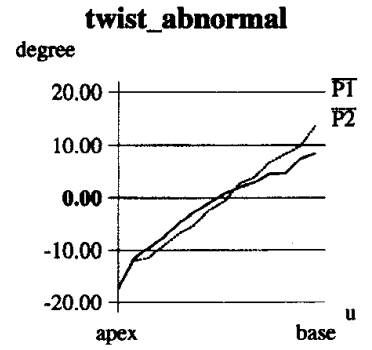

(b)
Fig. 15. Extracted LV parameter $\tau(u)$ at end-systole: (a) normal hearts, and (b) abnormal hearts.

characterize a detailed motion of the deforming model along its long axis and over time.

We applied our technique to another normal LV to verify the result and we found that the extracted parameter functions were very similar to those shown in Fig. 10. The overall contraction was approximately $25 \%$. Like in the other normal $\mathrm{LV}$, there was less contraction (defined by $a_{2}$ ) along the $y$ axis toward the base of the LV compared with the contraction toward the apex. The second normal heart was known to have greater twisting motion during systole. Surprisingly, we found that the LV underwent greater global rotational motion initially, before twisting. Fig. 15(a) shows the twisting parameters at the end-systole for both normal LV's. The twisting angle during systole was quantified to be approximately $20^{\circ}$.

2) Abnormal LV's in Systole: To further evaluate our model fitting technique, we also fit our model to abnormal heart data from two patients with hypertrophic cardiomyopathy. Abnormal hearts with hypertrophic cardiomyopathy are generally bigger in size, but do not pump as well as normal hearts. While the results were similar for these two abnormal hearts, they were different from those we obtained for the normal hearts. Fig. 11 shows the fitted models to one 


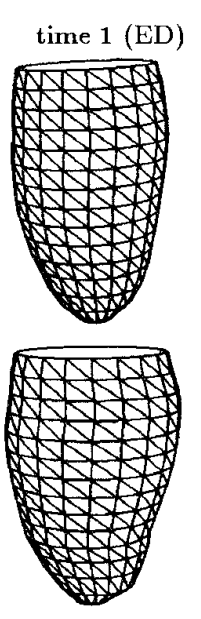

(a)

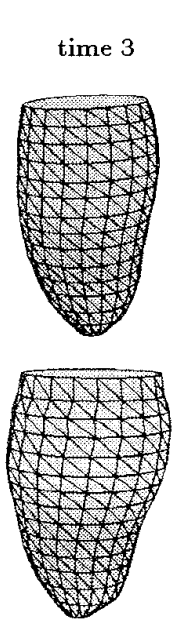

parameter $a_{1}(u)$

time 4 time $5(\mathrm{ES})$
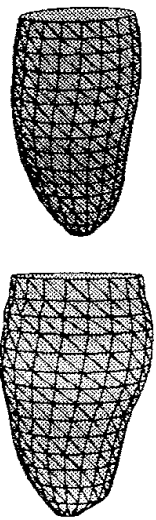

(b)

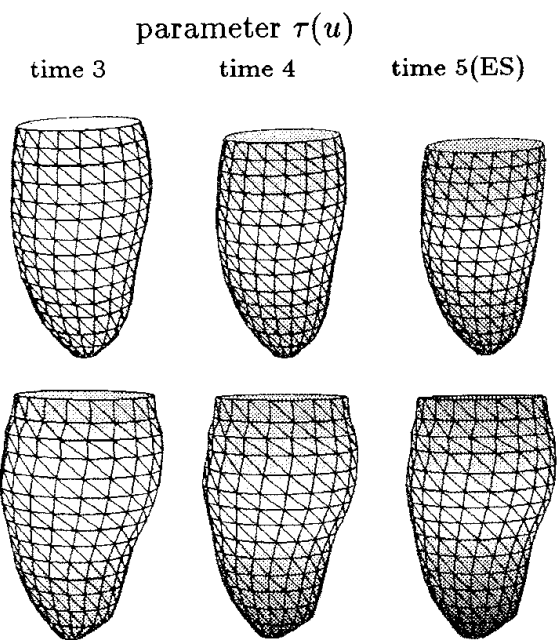

(c)

Fig. 16. Visualizing changes of values in parameter functions. (Darker drawn areas indicate greater deviation from the initial state.)

of the abnormal heart data, while Fig. 12 shows graphs of the model's parameter functions which may be compared with the normal heart's model parameter functions, shown in Fig. 10. The radial contraction of the abnormal heart is approximately $15-20 \%$, and the longitudinal contraction is approximately $7 \% \%^{6}$. Note that the overall contraction decreases significantly toward the apex. The twisting motion during its systole is approximately $27^{\circ}$. Finally, from the graphs shown in Fig. 12(e) and (f), we observe that there is greater long-axis deformation compared with the normal LV's.

\section{Comparing Normal and Abnormal LV Parameters}

The top row of Fig. 13 shows four fitted models at the end-diastole: the first two are of healthy volunteers (V1, V2), and the other two are of patients (P1, P2) with hypertrophic cardiomyopathy. The second row of Fig. 13 shows the corresponding LV at the end-systole. As shown in the figure, the LV's with hypertrophic cardiomyopathy are bigger in size than the normal LV's. Figs. 14 and 15 show plots of extracted parameters at end-systole for two normal, and two abnormal LV's. The extracted parameter functions $a_{1}(u)$ (shown in Fig. 14), where the radial contraction of a heart is captured, show that the abnormal hearts contract much less than the normal hearts, especially toward the apex. The extracted twisting parameter functions (shown in Fig. 15), however, show that the abnormal hearts twist more than the normal hearts. It seems that the abnormal hearts twist more, perhaps to compensate for their inability to contract as much as a normal heart. As we plotted the graphs of extracted parameters from the fitting process, not only can we observe the results qualitatively, but we can also measure the changes quantitatively. Therefore, we are able to quantitatively verify a result about the above abnormal hearts that was qualitatively evident to physicians.

\footnotetext{
${ }^{6}$ Total displacement along the $z$ axis is approximately $7 \mathrm{~mm}$ where the normalized length of the LV is $107 \mathrm{~mm}$.
}

Note that all the model fitting results are within an acceptable error bound, since rms errors are less than $0.5 \mathrm{~mm}$, where the length of the hearts is approximately $100 \mathrm{~mm}$.

Visualizing Extracted Parameter Functions: In order to view the changes in the parameters during systole, we can shade the model $^{7}$ with respect to a certain parameter, as shown in Fig. 16(b) and (c). The images in the first row of Fig. 16 are the recovered models from the first set of normal heart data at different time frames, and those in the second row are the recovered models from the first set of abnormal heart data. In Fig. 16(a) the models at the initial time frame are shaded in white. As the respective parameter value changes, the shading in the corresponding part of the model becomes darker. Fig. 16(b) and (c) shows the models shaded according to the values of the $a_{1}(u)$ (radial contraction) and the $\tau(u)$ parameters (twist) at times 3,4 , and 5 , respectively. It can be easily observed that, compared with the normal heart, the abnormal heart has less radial contraction at the end of systole [compare changes in shading in Fig. 16(b)], but more twisting motion [compare changes in shading in Fig. 16(c)]. We can also observe that the parameter variation between the two LV's is greater toward the apex.

Using this new family of primitives which are defined based on parameter functions, we can capture and quantify the $\mathrm{LV}$ motion and shape changes in an intuitive way. Therefore, we can quantitatively compare normal and abnormal hearts and present the results in a way that can be readily understood by physicians.

\section{CONCLUSION}

We have presented a new class of physics-based deformable models that can be used in many applications, among them the very important problem of analyzing the shape and motion of the LV from tagged MRI data. The significant aspect of

\footnotetext{
${ }^{7}$ Even though we show shaded models, the computer screen representation of value changes in the parameter variation is based on the use of different colors in addition to shading.
} 
these new models is that their global parameters are functions allowing the representation of complex shapes with a few intuitive parameters. For the applications in this paper, we were able to eliminate the need for calculation of local deformation parameters that require nontrivial processing to provide a compact and intuitive representation of shape. We demonstrated the applicability of our technique to the shape and motion analysis of the mid-wall of the LV for normal and abnormal hearts during systole, from tagged data. By plotting the parameter functions over time we were able to make comparisons between normal and abnormal hearts and verify quantitatively, qualitative knowledge about the LV motion known to physicians. We plan to apply our framework to multiple normal and abnormal hearts to be able to quantitatively characterize the ranges of normal LV motion and the effects of the various LV diseases to the LV shape and motion.

\section{ACKNOWLEDGMENT}

The authors would like to thank K. Fellows, Director of the Department of Radiology at the Children's Hospital of Philadelphia, PA for supporting the work of J. Park.

\section{REFERENCES}

[1] T. Arts, W. C. Hunter, A. Douglas, M. M. Muijtjens, and R. S. Reneman, "Description of the deformation of the left ventricle by a kinematic model," J. Biomech., vol. 25, no. 10, pp. 1119-1127, 1992.

[2] L. Axel and L. Dougherty, "Heart wall motion: Improved method of spatial modulation of magnetization for MR imaging," Radiol, vol. 172, pp. 349-350, 1989.

[3] A. Amini and J. Duncan, "Pointwise tracking of left-ventricular motion in 3-D," in Proc. IEEE Workshop on Visual Motion, Princeton, NJ, 1991, pp. 294-298.

[4] E. Bardinet, N. Ayache, and L. D. Cohen, "Fitting of iso-surfaces using superquadrics and free-form deformations," in Proc. of IEEE Workshop on Biomedical Image Analysis, Seattle, WA, June 1994, pp. 184-193.

[5] A. H. Barr, "Superquadrics and angle-preserving transformations," IEEE Comput. Graphics, Applicat., vol. 1, pp. 11-23, 1981.

[6] R. Beyar and S. Sideman, "Effect of the twisting motion on the nonuniformities of transmural fiber mechanics and energy demands-A theoretical study," IEEE Trans. Biomed. Eng., vol. BME-32, pp. 764-769, 1985.

[7] P. Burger and D. Gillies, Interactive Computer Graphics. Wokingham, England: Addison-Wesley, 1989.

[8] C. W. Chen and T. S. Huang, "Epicardial motion and deformation estimation from coronary artery bifurcation points," in Proc. 3 rd Int. Conf. Comput. Vision, Osaka, Japan, 1990, pp. 456-459.

[9] L. D. Cohen and I. Cohen, "A finite element method applied to new active contour models and 3-D reconstruction from cross sections," in Proc. 3 rd Int. Conf. Comput. Vision, Osaka, Japan, 1990, pp. 587-591.

[10] T. S. Denney Jr. and J. L. Prince, "3-D displacement filed reconstruction on an irregular domain from planar tagged cardiac MR images," in Proc. Workshop on Motion of Non-Rigid and Articulated Objects, Austin, TX, 1994, pp. 172-177.

[11] D. Friboulet, I. E. Magnin, and D. Revel, "Assessment of a model for overall left-ventricular three-dimensional motion from MRI data," Int J. Cardiac Imag., vol. 8, pp. 175-190, 1992.

[12] W. C. Huang and D. Goldgof, "Adaptive-size meshes for rigid and nonrigid shape analysis and synthesis," IEEE Trans. Pattern Anal., Machine Intell., vol. 15, no. 6, pp. 611-616, 1993.

[13] H. C. Kim, B. G. Min, M. M. Lee, J. D. Seo, Y. W. Lee, and M. C. Han, "Estimation of local cardiac wall deformation and regional wall stress from biplane coronary cineangiograms," IEEE Trans. Biomed. Eng., vol. BME-32, pp. 503-511, 1985.

[14] D. Marr and K. Nishihara, "Representation and recognition of the spatial organization of three-dimensional shapes," in Proc. Roy. Soc. London B, 1978.
[15] T. McInerney and D. Terzopoulos, "A finite element model for 3-D shape reconstruction and nonrigid motion tracking," in Proc. 4th Int. Conf. Comput. Vision, Berlin, Germany, 1993, pp. 518-523.

[16] D. Metaxas, "Physics-based modeling of nonrigid objects for vision and graphics, Ph.D. thesis, Department of Computer Science, University of Toronto, 1992.

[17] D. Metaxas and D. Terzopoulos, "Shape and nonrigid motion estimation through physics-based synthesis," IEEE Trans. Pattern Anal. Machine Intell., vol. 15, no. 6, pp. 569-579, 1993.

[18] S. K. Mishra and D. B. Goldgof, "Motion analysis and modeling of epicardial surfaces from point and line correspondences," in Proc. IEEE Workshop on Visual Motion, Princeton, NJ, 1991, pp. 300-305.

[19] C. Moore, W. O'Dell, E. McVeigh, and E. Zerhouni, "Calculation of three-dimensional left-ventricular strains form biplanar tagged MR images," J. Mag. Res. Imag., vol. 2, pp. 165-175, 1992.

[20] C. Nastar and N. Ayache, "Spatio-temporal analysis of nonrigid motion from 4-D data," in Proc. Workshop on Motion of Non-Rigid and Articulated Objects, Austin, TX, 1994, pp. 146-151.

[21] J. Park, D. Metaxas, and A. Young, "Deformable models with parameter functions: Application to heart-wall modeling," in Proc. IEEE Computer Vision and Pattern Recog., Seattle, WA, 1994, pp. 437-442.

[22] J. Park, D. Metaxas, A. Young, and L. Axel, "Model-based analysis of cardiac motion from tagged MRI data," in Proc. 7th Annu. IEEE Symp. Computer-Based Med. Syst., Winston-Salem, North Carolina, 1994, pp. $40-45$.

[23] J. Park, D. Metaxas, and L. Axel, "Volumetric deformable models with parameter functions: A new approach to the 3-D motion analysis of the LV from MRI-SPAMM," in Proc. 5th Int. Conf. Comput. Vision, Cambridge, MA, 1995, pp. 700-705.

[24] "Analysis of left-ventricular wall motion based on volumetric deformable models and MRI-SPAMM," Med. Image Anal. J., vol. 1, no. 1, pp. 1-19, 1996.

[25] A. Pentland and S. Sclaroff, "Closed-form solutions for physically based shape modeling and recognition," IEEE Trans. Pattern Anal. Machine Intell., vol. 13, no. 7, pp. 715-729, 1991.

[26] W. H. Press, B. P. Flannery, S. A. Teukolsky, and W. T. Vetterling, Numerical Recipes in C: The Art of Scientific Computing. Cambridge, UK, Cambridge Univ. Press, 1988.

[27] W. Rogers, E. Shapiro, J. Weiss, M. Buchalter, F. Rademakers, M Weisfeldt, and E. Zerhouni, "Quantification of and correction for leftventricular systolic long-axis shortening by magnetic resonance tissue tagging and slice isolation," Circ., vol. 84, pp. 721-731, 1991.

[28] S. Sclaroff and A. Pentland, "On modal modeling for medical images: Underconstrained shape description and data compression," in Proc. IEEE Workshop Biomed. Image Anal., Seattle, WA, 1994, pp. 70-79.

[29] T. W. Sederberg and S. R. Parry, "Free-form deformation of solid geometric models," in Proc. SIGGRAPH, Comput. Graphics, Dallas, TX, 1986, vol. 20, no. 4, pp. 151-160.

[30] A. A. Shabana, Dynamics of Multibody Systems. New York: WileyInterscience, 1988.

[31] P. Shi, A. Amini, G. Robinson, A. Sinusas, C. T. Constable, and J. Duncan, "Shape-based 4-D left-ventricular myocardial function analysis," in Proc. IEEE Workshop Biomed.Image Anal., Seattle, WA, 1994, pp. $88-97$.

[32] R. Tennant and C. J Wiggers, "The effect of coronary occlusion on myocardial contraction," Am. J. Physiol, vol. 112, pp. 351-361, 1935.

[33] A. Pentland and B. Horowitz, "Recovery of nonrigid motion and structure," IEEE Pattern Anal. Machine Intell., vol. 13, no. 7, pp. $730-742,1991$.

[34] D. Terzopoulos and D. Metaxas, "Dynamic 3-D models with local and global deformations: Deformable superquadrics," IEEE Trans. Pattern Anal. Machine Intell., vol. 13, no. 7, pp. 703-714, 1991.

[35] S. M. Nidorf and A. E. Weyman, "Left ventricle II: Quantification of segmental dysfunction," in Principles and Practice of Echocardiography, A. E. Weyman, Ed. Philadelphia, PA: Lea and Febiger, 1994, pp. $625-655$.

[36] A. A. Young and L. Axel, "Three-dimensional motion and deformation of the heart wall," Radiol, vol. 185, pp. 241-247, 1992.

[37] "Non-rigid heart wall motion using MR tagging," in Proc. IEEF Conf. Comput. Vision Pattern Recognition, 1992, pp. 399-404.

[38] A. A. Young, D. L. Kraitchman, L. Dougherty, and L. Axel, "Tracking and finite element analysis of stripe deformation in magnetic resonance tagging," IEEE Trans. Med. Imag., vol. 14, no. 3, pp. 413-421, 1995.

[39] E. A. Zerhouni, D. M. Parish, W. J. Rogers, and A. Yang, "Human heart: Tagging with MR imaging-A method for noninvasive assessment of myocardial motion," Radiol., vol. 169, pp. 59-63, 1988. 